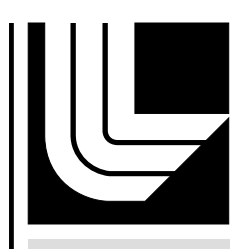

LAWRENCE LIVERM ORE NATIONAL LABORATORY

Non-LTE Modeling for the National Ignition Facility (and Beyond)

H. A. Scott, B. A. Hammel, S. B. Hansen

July 13,2011

The 17th International Conference on Atomic Processes in Plasmas Belfast, United Kingdom July 19, 2011 through July 22, 2011 
This document was prepared as an account of work sponsored by an agency of the United States government. Neither the United States government nor Lawrence Livermore National Security, LLC, nor any of their employees makes any warranty, expressed or implied, or assumes any legal liability or responsibility for the accuracy, completeness, or usefulness of any information, apparatus, product, or process disclosed, or represents that its use would not infringe privately owned rights. Reference herein to any specific commercial product, process, or service by trade name, trademark, manufacturer, or otherwise does not necessarily constitute or imply its endorsement, recommendation, or favoring by the United States government or Lawrence Livermore National Security, LLC. The views and opinions of authors expressed herein do not necessarily state or reflect those of the United States government or Lawrence Livermore National Security, LLC, and shall not be used for advertising or product endorsement purposes. 


\title{
Non-LTE Modeling for the National Ignition Facility (and Beyond)
}

\author{
H. A. Scott ${ }^{\mathrm{a}}$, B. A. Hammel ${ }^{\mathrm{a}}$ and S. B. Hansen ${ }^{\mathrm{b}}$ \\ ${ }^{a}$ Lawrence Livermore National Laboratory, PO Box 808, Livermore, CS 94550, USA \\ ${ }^{b}$ Sandia National Laboratories, PO Box 5800, Albuquerque, NM 87185, USA
}

\begin{abstract}
Considerable progress has been made in the last year in the study of laser-driven inertial confinement fusion at the National Ignition Facility (NIF). Experiments have demonstrated symmetric capsule implosions with plasma conditions approaching those required for ignition. Improvements in computational models - in large part due to advances in non-LTE modeling - have resulted in simulations that match experimental results quite well for the X-ray drive, implosion symmetry and total wall emission [1].
\end{abstract}

Non-LTE modeling is a key part of the NIF simulation effort, affecting several aspects of experimental design and diagnostics. The $\mathrm{X}$-rays that drive the capsule arise from high- $\mathrm{Z}$ material ablated off the hohlraum wall. Current capsule designs avoid excessive preheat from high-energy X-rays by shielding the fuel with a mid-Z dopant, which affects the capsule dynamics. The dopant also mixes into the hot spot through hydrodynamic instabilities, providing diagnostic possibilities but potentially impacting the energy balance of the capsule [2]. Looking beyond the NIF, a proposed design for a fusion reactor chamber depends on lowdensity high- $Z$ gas absorbing $\mathrm{X}$-rays and particles to protect the first wall [3].

These situations encompass a large range of temperatures, densities and spatial scales. They each emphasize different aspects of atomic physics and present a variety of challenges for nonLTE modeling. We discuss the relevant issues and summarize the current state of the modeling effort for these applications.

Keywords: NLTE, laser fusion

PACS: 52.20, 52.57.-z

\section{INTRODUCTION}

Non-LTE modeling has long been used in the ICF community. Until a couple years ago, the workhorse method was the screened-hydrogenic average-atom model, which attempts to represent all atomic processes occurring within a given atom with a single average ionization stage consisting of a handful of states. More recent simulations for NIF use the DCA model, which better represents the atomic physics occurring in the hot high-Z plasma inside a hohlraum. In particular, DCA includes a reasonable treatment of autoionization and dielectronic recombination processes, which are very difficult to handle with an average-atom treatment. DCA is a critical part of the "highflux model" (HFM) now routinely used for NIF simulations. 
Although DCA stands for "detailed configuration accounting", the atomic model used in these simulations is based upon superconfigurations. In the context of these integrated simulations, DCA denotes both the computational package used to simulate NLTE physics and the particular data used in those simulations. The name refers to the capability of the package to use detailed data, but these simulations use highly averaged atomic models generated using a screened-hydrogenic formalism, simple expressions for transition rates and a small amount of tabulated data. The construction of these models and generation of the data is described in detail in [4]. Atomic model generation is quite fast and flexible, allowing for easy experimentation with numbers of singly-, doubly-, or even triply-excited states or inner-shell holes on a sequence-bysequence basis. These simulations use a standard construction that includes enough structure to model physical processes and spectral features over a very wide energy range without excessive simulation costs.

This highly averaged atomic model stands in sharp contrast to the detailed models that can now be constructed and used for spectroscopic calculations. Use of these detailed models tends to be limited by computational cost. Simulations of NIF hohlraums and capsules necessarily include a wide range of materials, conditions and physical processes, making it infeasible to incorporate detailed atomic models in these integrated simulations. However, the DCA models used here have benefited greatly from comparisons to detailed models, and from the improved understanding of NLTE modeling developed over the last several years (see [5] and references therein).

Although these DCA models have been sufficiently accurate, and very productive, for hohlraum simulations, shortcomings of this approach become apparent in the context of different applications. In this paper, we discuss the use of DCA in three applications related to NIF.

\section{HOHLRAUM SIMULATIONS}

The primary application of NLTE physics in simulations of indirect-drive laser targets involves the overall energy balance in a hohlraum. Laser-heated high-Z material $(\mathrm{Au})$ expands off the hohlraum wall and is heated to temperatures of several $\mathrm{keV}$. For NIF-scale hohlraums, the energy required for producing this plasma, and emitted by this plasma, is a very significant fraction $(>30 \%)$ of the total energy budget. Accurate representation of this plasma is then critical to the overall simulation effort. The previous computational method, using an average-atom model, retained too much energy within the plasma, producing much higher temperatures and a correspondingly lower X-ray flux within the hohlraum than the current DCA-based method. The difference is largest for an empty hohlraum, as displayed in Figure 1, which compares simulations of the radiative flux with experimental data from one of the first NIF hohlraum shots in 2009 [6]. 


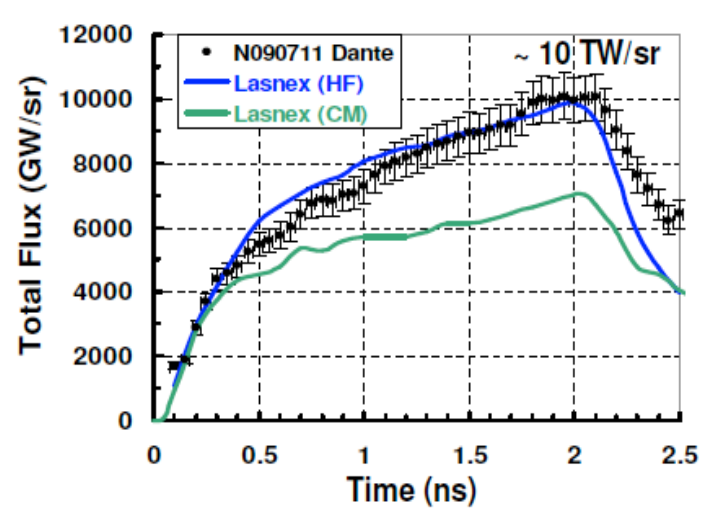

FIGURE 1. X-ray flux for scale 0.7 vacuum hohlraum. Solid lines give simulated results using the HFM (upper curve) and configuration managed (CM) model (lower curve). Dante measurements are given by points with error bars

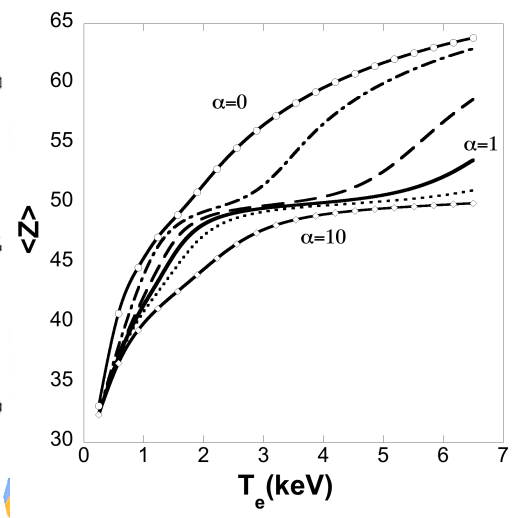

FIGURE 2. Average ionization state for $\mathrm{Au}$ as a function of $\mathrm{T}_{\mathrm{e}}$ with $\mathrm{n}_{\mathrm{e}}=1 \times 10^{21} \mathrm{~cm}^{-3}$ for several different values of $\alpha$. Solid line w/diamonds: $\alpha=10$; dotted: $\alpha=2$; solid: $\alpha=1$; dashed: $\alpha=0.5$; dash-dotted: $\alpha=0.1$, and solid w/circles: $\alpha=0$.

The large difference between the simulations was primarily due to the lack of autoionization / dielectronic recombination (AI/DR) in the average-atom model, resulting in a much more highly ionized plasma than that predicted by DCA. (An option for AI/DR did exist in the average-atom model, but was unreliable and vastly overestimated these processes). Although this is a complex atomic system with 40+ bound electrons, the treatment used in DCA appears to be adequate. Fortunately, although the AI/DR processes must be present, high accuracy is not required. Figure 2 shows the average ion charge $\langle\mathrm{Z}>$ as a function of temperature for Au plasma with an electron density of $10^{21} \mathrm{~cm}^{-3}$, characteristic of hohlraum coronal plasma. Each curve was calculated with a multiplier $\alpha$ applied to the AI/DR rates, with $\alpha=0,0.1,0.5,1$, 2 , and 10. Particularly in the relevant temperature range of $2-3 \mathrm{keV}$, the results are quite insensitive to the value of $\alpha$. Other quantities, such as the M-band radiation discussed below, are equally insensitive [7].

\section{DOPED CAPSULES}

Along with the effect on hohlraum energy balance, the Au coronal plasma also produces a significant amount of radiation with photon energies in the $2-4 \mathrm{keV}$ range, corresponding to transitions in the M-shell of Au. This M-band radiation can penetrate through the low- $Z$ capsule shell, preheating the fuel and degrading the implosion characteristics. To eliminate this source of preheating, the capsule shell is seeded with a mid- $Z$ dopant that absorbs the $M$-band radiation. The current NIF capsule design calls for a $\mathrm{CH}$ shell with Ge doping. The amount of Ge varies with position in a manner designed to reduce hydrodynamic instabilities, with the maximum being $1 \%$ by number density.

NLTE modeling of the dopant would be unnecessary, were it not for the hydrodynamic instabilities. Growth of perturbations on material interfaces - primarily those between the fuel and ablator and on the outside of the ablator - can mix shell material into the fuel. Increased emission from this material will reduce the 
temperature of the hot spot, preventing ignition if the injected mass is too large [8]. An LTE treatment of the Ge component of this material overstates the additional emission by a factor of two, despite the small concentration of Ge, so DCA is used for this aspect of the simulation. In addition, the presence of $\mathrm{Ge}$ in this material raises the possibility of a spectroscopic diagnostic for the mixed material. This is discussed in detail elsewhere, for simulations [2] and for application to experimental data [8]. Here we consider the implications and requirements for NLTE modeling of observed spectrum.

Figure 3 shows the innermost portion of the results of a 2D simulation of a Rev 5 capsule (see [2] for details). The simulation follow the evolution of a perturbation due to the fill tube, shown here at a time several ps before peak compression. The lefthand side of the figure shows material regions, while the right-hand side shows density. Temperatures of the mixed material range from $3-5 \mathrm{keV}$, with the hot spot reaching a peak temperature close to $6 \mathrm{keV}$.

The standard DCA model does not include spectroscopic detail, so spectral calculations use an atomic model with data for H-like through B-like sequences calculated with FAC [10]. These sequences are averaged to superconfigurations for principal quantum numbers above four, and for all principal quantum numbers for the B-like sequence, matching the structure of the equivalent DCA sequence. The standard DCA model is used for sequences with more than 5 bound electrons. This construction provides an atomic model that includes all sequences, and has spectroscopic detail for K-shell transitions, including many satellites.

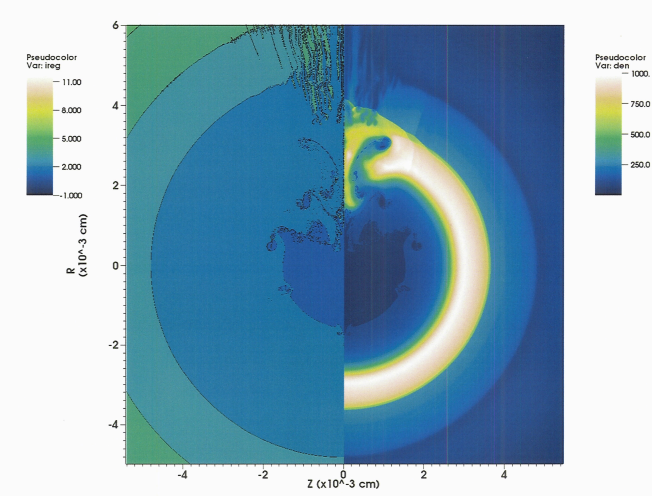

FIGURE 3. 2D simulation of Rev 5 THD several ps before peak compression. The left side shows material regions and the right side shows mass density. The defect on the pole (axis of symmetry) is due to the fill tube.

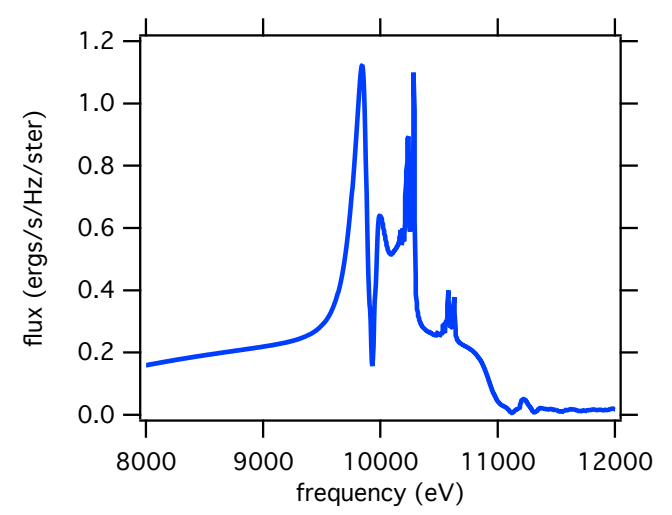

FIGURE 4. Simulated X-ray spectrum from capsule of Fig. 3. Emission is integrated over central $100 \mu \mathrm{m} \times 100 \mu \mathrm{m}$ area but is instantaneous in time.

Figure 4 shows the spectrum obtained from this simulation, integrated over a 100 $\mu \mathrm{m}$ by $100 \mu \mathrm{m}$ area centered on the capsule. The spectrum displays a number of interesting features. As expected, K-shell emission appears around 10.2 and $10.6 \mathrm{keV}$, corresponding to He- $\alpha$ and Ly- $\alpha$. For this time-resolved spectrum, the emission is primarily due to these transitions, but in time-integrated spectra the red components dominate and mostly come from Li-like and Be-like satellites. These emission features, corrected for optical depth effects, can be used to approximately infer the 
amount of hot $\mathrm{Ge}$ in the fuel region [2,9]. A large emission feature also appears at energies between 9.5 and $10.0 \mathrm{keV}$. This is a blend of $2 \mathrm{p} \rightarrow 1 \mathrm{~s}$ fluorescence features from cold ( $200 \mathrm{eV}$, M-shell) Ge in the ablator, pumped by radiation from the hot spot. Slightly warmer (300 eV, L-shell) Ge produces $1 \mathrm{~s} \rightarrow 2 \mathrm{p}$ absorption, also visible in the spectrum.

The challenge for NLTE modeling here arises from the wide range of conditions that must be considered. Material at temperatures from below $100 \mathrm{eV}$ up to several $\mathrm{keV}$, and densities up to $1000 \mathrm{~g} / \mathrm{cc}$ contribute to the implosion physics and observed spectrum. High optical depths, in lines as well as continua, require radiation transport treatments, while very large densities stress models for line broadening and continuum lowering.

\section{THE LIFE CHAMBER}

The Laser Inertial Fusion Energy (LIFE) effort is focused on providing a laser fusion power plant [11]. The target chamber is an important component of this system, providing a suitable environment for repetitive shots (10-15 Hz) over a lengthy operating period. The current design calls for the chamber to be filled with low-density $\left(6 \mu \mathrm{g} / \mathrm{cm}^{3}\right)$ Xe gas to absorbs X-rays and charged particles from the igniting capsules, protecting the first wall from large temperature spikes. The gas must then reset before the next shot to conditions permitting injection of the next target and propagation of laser beams.

Key simulation goals are determining the evolution of the gas between shots, and over the course of multiple shots, using NLTE physics combined with radiation transport, hydrodynamics and other processes. The temperature and ionization balance of the Xe are of particular interest, as they directly affect hydrodynamic motion of the gas and laser propagation.

Here, we focus on NLTE simulations of the Xe gas, starting with the response to the prompt X-ray flash. Figure 5 shows the electron temperature spatial profile $10 \mathrm{~ns}$ after an $8 \mathrm{MJ} \mathrm{X}$-ray flash, with temperatures close to the target reaching several hundred $\mathrm{eV}$. The gas near the wall is unaffected by the flash, due to absorption by the intervening gas and geometrical dilution of the flux. Evolution after the flash is strongly affected by radiation trapping, which lasts for some $10 \mathrm{~s}$ to $100 \mathrm{~s}$ of $\mu \mathrm{s}$.

To analyze the impact of the atomic physics after most of the radiation has escaped, we consider the evolution of optically thin Xe plasma with a starting temperature of $100 \mathrm{eV}$. Figure 6 shows the time-dependence of the electron and ion temperatures starting from this initial condition as calculated with DCA. Radiative recombination cools the electrons quickly, on timescales of order $10 \mu \mathrm{s}$, while the ions cool via coupling to the electrons. As the temperature drops below $\sim 1 \mathrm{eV}$ and the Xe becomes nearly neutral, the ions decouple from the electrons. Further cooling of the gas occurs only through much weaker atom-atom interactions, effectively only on much longer timescales that those of interest here. The final gas temperature is determined by the interplay of atomic processes and electron-ion coupling.

Modeling the atomic physics of (near-) neutral species requires more detail than principal quantum number superconfigurations (or the periodic table would look quite different), so DCA results for the late-time behavior are suspect. For comparison 
purposes, we have calculated Xe properties using a model for $\mathrm{Xe}^{0}$ and $\mathrm{Xe}^{+}$constructed with detailed data from FAC. Figure 6 compares the radiative cooling rates for temperatures below $1 \mathrm{eV}$ obtained with this detailed model and with DCA. The detailed model used about 20000 levels to describe these two charge states, versus 32 levels for DCA, with a corresponding increase in computational cost. Extensions to DCA aimed at improving the description of near-neutral species are currently under consideration.

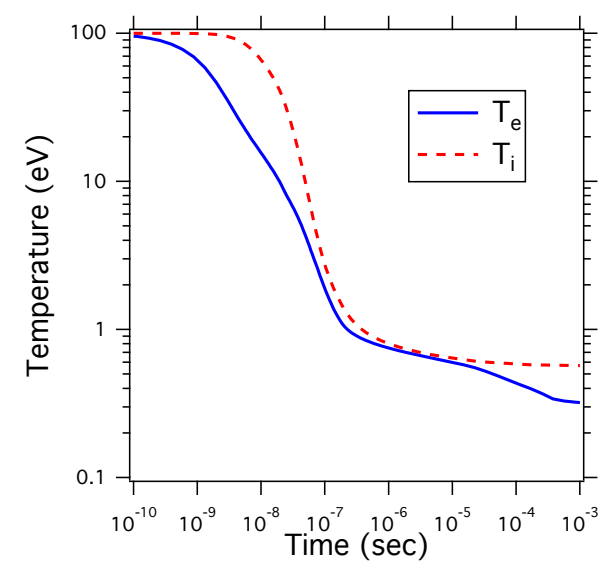

FIGURE 5. Electron (solid) and ion (dashed) temperature as a function of time for optically thin $6 \mu \mathrm{g} / \mathrm{cm}^{3} \mathrm{Xe}$, as calculated with DCA.

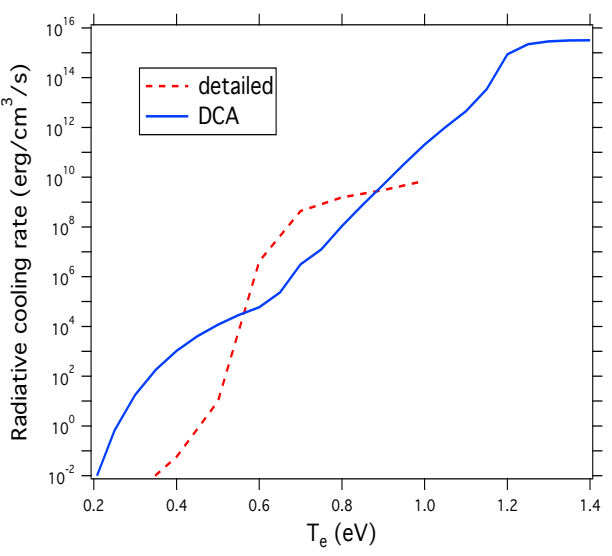

FIGURE 6. Radiative cooling rate as a function of electron temperature for optically thin $6 \mu \mathrm{g} / \mathrm{cm}^{3} \mathrm{Xe}$, as calculated with DCA (solid) and with a detailed model (dashed).

\section{ACKNOWLEDGMENTS}

This work performed under the auspices of the U.S. Department of Energy by Lawrence Livermore National Laboratory under Contract DE-AC52-07NA27344. SH was supported by Sandia National Laboratories, a multiprogram laboratory operated by Sandia Corporation, a Lockheed Martin Company, for the U.S. Department of Energy under Contract No. DE-AC04-94AL85000.

\section{REFERENCES}

1. M. D. Rosen, H. A. Scott, D. E. Hinkel, et al, HEDP 7, 180-190 (2011).

2. B. A. Hammel, H. A. Scott, S. P. Regan, et al, Physics of Plasmas 18, 056310 (2011).

3. J. F. Latkowski, R. P. Abbot, S. Aceves, et al, Fusion Science and Technology 60, 54-60 (2011).

4. H. A. Scott and S. B. Hansen, HEDP 6, 39-47 (2010).

5. C. J. Fontes, J. Abdallah, Jr., C. Bowen, R. W. Lee and Yu. Ralchenko, HEDP 5, 15-22 (2009).

6. R. E. Olsen, L. J. Suter, J. L. Kline, et al, in "Inertial Fusion Sciences and Applications 2009”, B. Hammel, C. Laubanne, H. Azechi, editors. Journal of Physics, IOP Publishers (2009).

7. H. A. Scott, C. T. Whelan and S. H. Glenzer, submitted to HEDP.

8. S. W. Haan, J. D. Lindl, D. A. Callahan, et al, Phys. Plasmas, in press.

9. S. P. Regan, this volume.

10. M. F. Gu, Astrophys. J. 590, 1131-1140 (2003).

11. E. Moses, T. Diaz De La Rubia, J. F. Latkowski, et al, Fusion Science and Technology 56, 566-572 (2009). 